\title{
Direct vancomycin irrigation as treatment for resistant aortic bed infection
}

\author{
Hannah Winter, Isaac Nyamekye
}

\begin{abstract}
Introduction: A case report of direct vancomycin irrigation as management of retroperitoneal abscess following removal of infected aortic graft. Case Report: A 63-yearold man presented nine years following aortic aneurysm repair with recurrence of aneurysm. At re-operation, aortic graft infection was diagnosed and managed with graft excision, debridement, axillo-bifemoral bypass and prolonged systemic antibiotics. The abscess failed to resolve. Direct vancomycin irrigation via percutaneous drainage catheter led to resolution of infection. Conclusion: There is little evidence to support vancomycin irrigation as treatment after removal of infected aortic grafts. Such an approach may be considered in patients failing to respond to traditional therapy.
\end{abstract}

Keywords: Vancomycin, Irrigation, Graft infection, Aneurysm, Aortic graft

$* * * * * * * * *$

Winter H, Nyamekye I. Direct vancomycin irrigation as treatment for resistant aortic bed infection.

Hannah Winter ${ }^{1}$, Isaac Nyamekye ${ }^{1}$

Affiliations: ${ }^{1}$ Department of Vascular Surgery, Worcester Royal Hospital, Worcestershire, UK.

Corresponding Author: Miss Hannah Winter, 48 West Street, Stourbridge, West Midlands, DY8 1XN, UK; Email: hwinter@doctors.org.uk

Received: 22 January 2011

Accepted: 25 March 2011

Published: 01 July 2011
International Journal of Case Reports and Images 2011;2(7):1-3.

$$
* * * * * * * * *
$$

doi:10.5348/ijcri-2011-07-41-CR-1

\section{INTRODUCTION}

Prosthetic graft infection is a recognised complication of aortic aneurysm surgery carrying a high morbidity and mortality [1]. Treatment of prosthetic graft infection has traditionally included excision of the infected prosthesis, extra-anatomical bypass and prolonged treatment with systemic antibiotics. We report a novel approach of treating persistent retroperitoneal abscess formation complicating aortic graft infection.

\section{CASE REPORT}

A 63-year-old man presented to his local accident and emergency department nine years following combined coronary bypass and infra-renal aortic aneurysm repair. He complained of abdominal pain radiating through to his back but remained hemodynamically stable. His comorbidites included a previous smoking habit, medicated hypertension and angina. Examination revealed a tender large aortic aneurysm. An urgent Computed Tomography (CT) scan demonstrated a $9.5 \mathrm{~cm}$ infra-renal abdominal aneurysm with contrast outside of the old graft but within the sac (Figure 1). He was transferred to our vascular unit and taken to theatre for repair of a false aneurysm.

On opening the sac frank pus was encountered alongside the old Dacron graft and a disruption to the proximal anastomosis line. Pus was sent for microbiology, the aortic graft excised and the ends of 


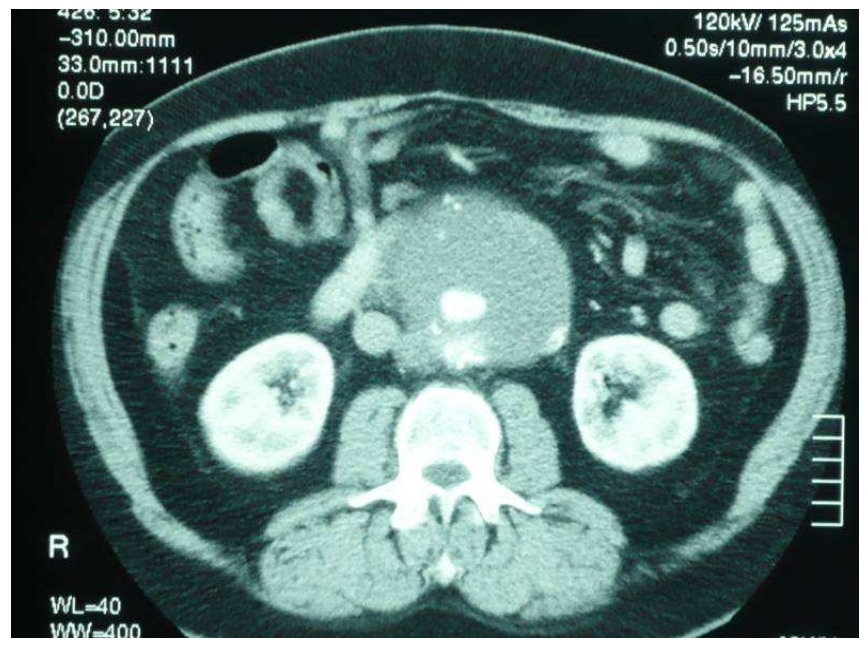

Figure 1: CT scan of false abdominal aortic aneurysm with contrast outside of the graft nine years following surgery for abdominal aortic aneurysm.

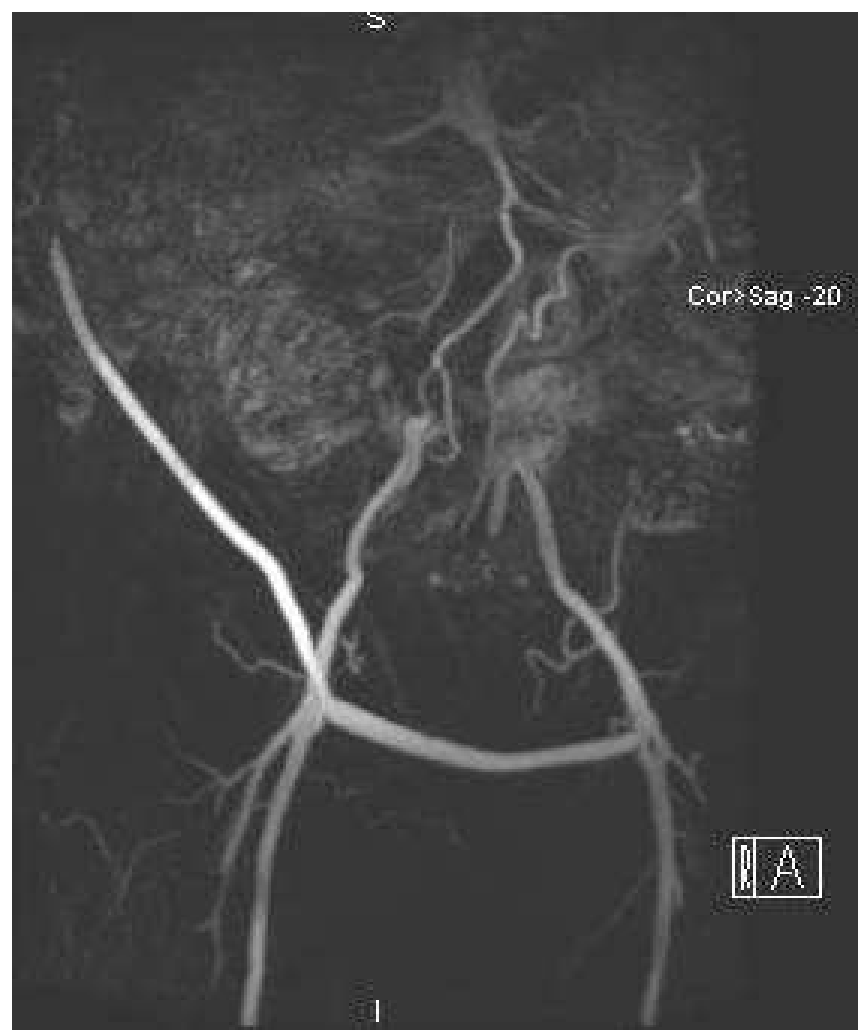

Figure 2: Magnetic Resonance Angiogram of axillobifemoral bypass grafting taken six years following formation.

native aorta over sewn. The aortic bed was radically debrided and an omental patch sutured to the anterior spinous ligament. An axillo-bifemoral bypass was placed from the right using a polytetrafluoroethylene (PTFE) graft (Figure 2). Perioperative vancomycin, cefuroxime and metronidazole were continued intravenously for two weeks.

No organisms were isolated from the pus contents. The patient made a good recovery and was discharged home on day 16 with a six week course of oral coamoxiclav and metronidazole following microbiology advice.

Abdominal ultrasonogram (USG) performed one month after discharge revealed no untoward abnormality. However, patient was re-admitted after two months with pyrexia of $39^{\circ} \mathrm{C}$, rigors, back pain and a raised C-reactive protein of $266 \mathrm{mg} / \mathrm{L}$, but a normal white cell count. An intravenous cocktail of cefuroxime, vancomycin, metronidazole and gentamicin was commenced on microbiology advice. CT imaging revealed a 6x4 7 $7 \mathrm{~cm}$ low attenuation area in the aortic bed suggestive of abscess formation. A white cell localisation scan was unremarkable. Turbid yellow retroperitoneal fluid was drained from the aortic bed under CT guidance. No organisms were isolated and blood cultures were negative.

Despite two weeks of intravenous antibiotics the patient continued to have spikes of high temperature and further imaging revealed recurrence of the retroperitoneal collection. A pigtail catheter was placed for drainage. After completion of drainage the catheter was used to instil $1 \mathrm{~g}$ of vancomycin diluted in $50 \mathrm{ml}$ $0.9 \%$ saline directly into the retroperitoneal space by placing the bag on a drip stand. The bag was clamped for two hours and then drained by placing it on the floor. As this was off-licence use of vancomycin, the treatment was personally performed by the senior author twice daily for nine days. The pyrexia settled and after catheter removal the patient made a full recovery. He was discharged home 48 hours later with lifelong Augmentin following microbiology advice. He initially was reviewed with USG six monthly for three years then annually and remains well after eight years with no recurrence of infection.

\section{DISCUSSION}

Aortic graft insertion is complicated by infection in $0.5-2 \%$ of cases [1]. Late graft infection typically differs from early infection by culturing more indolent bacteria as the infectious organism. Bacteria such as Staphylococcus epidermidis are difficult to eradicate and may chronically infect aortic grafts without causing any symptoms. Vancomycin has been shown to be a suitable antibiotic for infected prosthetic grafts due to its bactericidal properties and efficacy against Methicillin-resistant Staphylococcus aureus infections [1].

The successful use of a non-antibiotic agent, $0.5 \%$ povidone-iodine and super acidic solution to irrigate aortic bed infection has been documented in a vascular case [2]. Antibiotic irrigation itself has been documented as a treatment for septic arthritis with joint space lavage and also to treat postpneumonectomy empyema [3]. Schumaker and 
Mandelbaum used vancomycin and povidone-iodine irrigation to treat mediastinitis, reducing mortality from $31 \%$ to $1.6 \%$ when compared to intravenous vancomycin alone [4].

Morris and colleagues reported a prospective study in which 10 patients with infected aortic grafts were treated with antibiotic irrigation via silicone drainage tubes placed around anastomoses. They used gentamicin as the initial antibiotic and then altered the agent according to cultures with an irrigation time ranging from 12 to 56 days. The results were promising; six out of seven survivors were free from infection at four to seven years [5].

\section{CONCLUSION}

Intracavity vancomycin irrigation for recurrent aortic bed infection led to complete resolution of retroperitoneal sepsis in this case. We suggest that this technique could be a useful adjunct to surgical debridement for resistant retroperitoneal sepsis causing resolution of symptoms through local absorption of antibiotics and irrigation of surface bacteria.

\section{$* * * * * * * * *$}

\section{Author Contributions}

Hannah Winter - Substantial contributions to conception and design, Acquisition of data, Analysis and interpretation of data, Drafting the article, Revising it critically for important intellectual content, Final approval of the version to be published

Isaac Nyamekye - Substantial contributions to conception and design, Acquisition of data, Analysis and interpretation of data, Drafting the article, Revising it critically for important intellectual content, Final approval of the version to be published

\section{Guarantor}

The corresponding author is the guarantor of submission.

\section{Conflict of Interest}

There are no conflicts of interest.

\section{Copyright}

(C) Hannah Winter et al. 2011; This article is distributed under the terms of Creative Commons attribution 3.0 License which permits unrestricted use, distribution and reproduction in any means provided the original authors and original publisher are properly credited. (Please see www.ijcasereportsandimages.com /copyright-policy.php for more information.)

\section{REFERENCES}

1. Perera G, Fujitani R, Kubasha S. Aortic Graft Infection: Update on Management and Treatment
Options. Vasc Endovasc Surg. 2006;40(1):1-10.

2. Matsuda C, Sakakibara T, Sakagoshi N, Takano H. A Case of Successful Treatment for Graft Infection after Abdominal Aortic Aneurysm Repair. Japanese Journal of Cardiovascular Surgery. 2001;30(4):1879.

3. Ng T, Ryder B, Maziak D, Shamji F. Treatment of post-pneumonectomy empyema with debridement followed by continuous antibiotic irrigation. J Am Coll Surg. 2008;206(3):1178-83.

4. Schumacker H, Mandelbaum I. Continuous antibiotic irrigation in the treatment of infection. Arch Surg. 1963;86:384-7.

5. Morris G, Friend P, Vassallo D, Farrington M, Leapman S, Quick C. Antibiotic irrigation and conservative surgery for major aortic graft infection. J Vasc Surg. 1994;20(1):88-95. 